\title{
Insight into the effects of dexmedetomidine on intraoperative hemodynamics and postanesthetic recovery speed
}

\author{
Jaemin Lee \\ Department of Anesthesiology and Pain Medicine, Seoul St. Mary's Hospital, College of Medicine, Catholic University of Korea, Seoul, Korea
}

Dexmedetomidine is a potent alpha-2-adrenergic agonist, more selective than clonidine, with widespread actions that include anxiolysis, sedation, anesthetic-sparing, analgesia and sympatholytic properties. Since its release in the US market in late 1999, it has gained remarkable attention in the adult, pediatric and geriatric populations, predominantly because of its minimal respiratory depression. A large body of recent work supports its favorable profile in many other clinical scenarios, including neuroprotection, cardioprotection and renoprotection, with promising results [1].

Even though dexmedetomidine displays various favorable pharmacological actions, it is believed that its routine use on patients undergoing surgery is not the current trend. The reason for this can be summed up in two ways. First, intraoperative hemodynamic unstability including hypotension and/or bradycardia due to sympatholysis may be problematic in some patients. Such hemodynamic effects of dexmedetomidine may be expected to be more pronounced in hypovolemic patients and in those with diabetes mellitus or chronic hypertension and in the elderly. Secondly, a delay in postanesthetic recovery is expected due to dexmedetomine's relatively long duration of action compared to other short-acting anesthetics. This disadvantage means that dexmedetomidine is not suitable for day surgery patients.

An interesting study has been carried out associated with the effects of dexmedetomidine on intraoperative hemodynamics and postoperative recovery in this issue of Korean Journal of Anesthesiology [2]. Kang et al. [2] found that the changes in mean arterial pressure (MAP) during the operation in the dexmedetomidine group were significantly less than those in the control group, which means that intraoperative administration of dexmedetomidine produced more stable hemodynamics than in the control. In addition, the authors claimed that dexmedetomidine was not associated with prolongation of extubation time or compromised recovery profile. Although their study was small-scaled, with only 10 patients involved in each group, their results are interesting in that they presented some evidence to dispel our concerns about dexmedetomidine-induced intraoperative hypotension and delay in postanesthetic recovery. These concerns have made us reluctant to the routine use of dexmedetomidine in the operating room.

The hemodynamic effects of dexmedetomidine are somewhat controversial. Hypotension and/or bradycardia are not unusual, because dexmedetomidine decreases sympathetic nervous system activity. On the other hand, transient hypertension has been observed primarily during the loading dose in association with the initial peripheral vasoconstrictive effects, although treatment of transient hypertension has generally not been necessary. Investigations in both humans and animals have revealed significant cerebral vasoconstrictive effects of dexmedetomidine [3,4]. Also, dexmedetomidine is known to be associated with higher MAP than midazolam in patients under epidural anesthesia [5]. Such controversies regarding hemodynamic effect are thought to be caused by biphasic action of dexmedetomidine, characterized by an initial short-term increase in MAP followed by a longer lasting hypotension. The biphasic action of dexmedetomidine is sometimes displayed in a dose-dependent manner, i.e., lower dosages reduce norepinephrine release, resulting in decrease

Corresponding author: Jaemin Lee, M.D., Department of Anesthesiology and Pain Medicine, Seoul St. Mary's Hospital, Banpo-dong, 504, Seocho-gu, Seoul 137-701, Korea. Tel: 82-2-2258-6153, Fax: 82-2-537-1951, E-mail: jmlee@catholic.ac.kr

(c) This is an open-access article distributed under the terms of the Creative Commons Attribution Non-Commercial License (http:// creativecommons.org/licenses/by-nc/3.0/), which permits unrestricted non-commercial use, distribution, and reproduction in any medium, provided the original work is properly cited. 
in vascular tone and hypotension, and higher dosages produce alpha-2B-mediated vasoconstriction and hypertension [6]. One factor that should be remembered is that the hemodynamic effect of dexmedetomidine during anesthesia is, to some extent, implicated with its anesthetic sparing effect, because dexmedetomidine reduces the required amount of anesthetics necessary to maintain adequate depth of anesthesia.

Dexmedetomidine is basically a central nervous system depressant with a relatively long duration of action, and thus it causes delayed postanesthetic recovery. As was revealed in the Bührer et al. [7], dexmedetomidine reduced the thiopental dose requirement for electroencephalographic burst suppression by $30 \%$, which was not the result of a pharmacodynamic interaction but rather of a dexmedetomidine-induced decrease in thiopental distribution volume and distribution clearances. Also, dexmedetomidine decreases the BIS value itself [8]. These characteristics are considered to be responsible for delayed recovery from anesthesia after dexmedetomidine. On the other hand, some investigators reported that dexmedetomidine promoted an earlier emergence and reduced the length of stay in the postanesthetic care unit $[9,10]$. In these reports, they ascribed shorter recovery time to less amount of anesthetics needed when dexmedetomidine was co-administered.

On the whole, the Kang et al. [2] results of the stable intraoperative hemodynamics and the acceptable postanesthetic recovery speed in the dexmedetomidine group are thought to be related to the anesthetics-sparing effect of dexmedetomidine, not solely to the pharmacologic effect of dexmedetomidine itself. In their study, the Ce (effecter-site concentration) of TCIpropofol was controlled to the minimum dosage needed to maintain BIS of 45-55, while the Cp (plasma concentration) of TCI-remifentanil was fixed to be $10 \mathrm{ng} / \mathrm{ml}$ during the operation. They found that the mean infusion rate of propofol as supplement anesthetics was about $30 \%$ lower by coadministration of dexmedetomidene than in the control. At the end of surgery, the Ce of propofol in the dexmedetomidine group was relatively lower than that in the control group. As a result, less propofol administration was responsible for less propofol-induced vasodilatory effect, which in turn maintained relatively stable intraoperative MAP, offsetting dexmedetomidine-induced delayed recovery.

Again, it should be reemphasized that the favorable hemo- dynamics during anesthesia and the comparable speed of recovery after anesthesia is attributed to dexmedetomidine's anesthetics sparing effect. Nevertheless, for the anesthetic management, dexmedetomidine-induced anesthetics sparing is meaningful in that a fundamental concept of anesthetic management is to minimize the side effects of anesthetic drugs by administrating the lowest dose necessary to maintain an adequate depth of anesthesia while ensuring a rapid recovery.

\section{References}

1. Chrysostomou C, Schmitt CG. Dexmedetomidine: sedation, analgesia and beyond. Expert Opin Drug Metab Toxicol 2008; 4: 619-27.

2. Kang WS, Kim SY, Son JC, Kim JD, Muhammad HB, Kim SH, et al. The effect of dexmedetomidine on the adjuvant propofol requirement and intraoperative hemodynamics during remifentanil-based anesthesia. Korean J Anesthesiol 2012; 62: 113-8.

3. Drummond JC, Dao AV, Roth DM, Cheng CR, Atwater BI, Minokadeh A, et al. Effect of dexmedetomidine on cerebral blood flow velocity, cerebral metabolic rate, and carbon dioxide response in normal humans. Anesthesiology 2008; 108: 225-32.

4. Nakano T, Okamoto H. Dexmedetomidine-induced cerebral hypoperfusion exacerbates ischemic brain injury in rats. J Anesth 2009; 23: 378-84.

5. Kuzucuoğlu T, Bölükbaşioğlu I, Arslan G, Yücel E, Ayaz B. Comparison of the activity and reliability of intravenous administration of midazolam and dexmedetomidine on sedation levels under epidural anesthesia. Agri 2010; 22: 121-30.

6. Snapir A, Posti J, Kentala E, Koskenvuo J, Sundell J, Tuunanen H, et al. Effects of low and high plasma concentrations of dexmedetomidine on myocardial perfusion and cardiac function in healthy male subjects. Anesthesiology 2006; 105: 902-10; quiz 1069-70.

7. Bührer M, Mappes A, Lauber R, Stanski DR, Maitre PO. Dexmedetomidine decreases thiopental dose requirement and alters distribution pharmacokinetics. Anesthesiology 1994; 80: 1216-27.

8. Sano H, Doi M, Mimuro S, Yu S, Kurita T, Sato S. Evaluation of the hypnotic and hemodynamic effects of dexmedetomidine on propofol-sedated swine. Exp Anim 2010; 59: 199-205.

9. Nunes RR, Cavalcante SL. Influence of dexmedetomidine upon sevoflurane end-expiratory concentration. Evaluation by bispectral index, suppression rate and electroencephalographic power spectral analysis. Rev Bras Anestesiol 2002; 52: 133-45.

10. Tufanogullari B, White PF, Peixoto MP, Kianpour D, Lacour T, Griffin J, et al. Dexmedetomidine infusion during laparoscopic bariatric surgery: the effect on recovery outcome variables. Anesth Analg 2008; 106: 1741-8. 